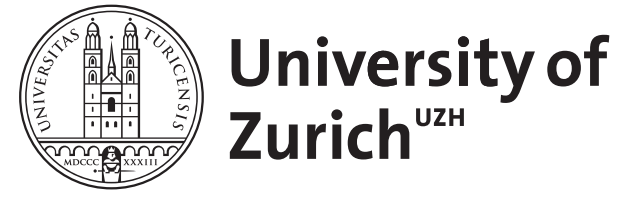

Zurich Open Repository and Archive

University of Zurich

University Library

Strickhofstrasse 39

CH-8057 Zurich

www.zora.uzh.ch

Year: 1993

Bestimmung der Liquorproduktionsrate mit Magnetresonanzmethoden

Botnar, René ; Scheidegger, Markus Beat ; Boesiger, Peter

DOI: https://doi.org/10.1515/bmte.1993.38.s1.347

Posted at the Zurich Open Repository and Archive, University of Zurich

ZORA URL: https://doi.org/10.5167/uzh-154348

Journal Article

Published Version

Originally published at:

Botnar, René; Scheidegger, Markus Beat; Boesiger, Peter (1993). Bestimmung der Liquorproduktionsrate mit Magnetresonanzmethoden. Biomedizinische Technik. Biomedical engineering, 38(s1):347-348.

DOI: https://doi.org/10.1515/bmte.1993.38.s1.347 


\title{
Bestimmung der Liquorproduktionsrate mit Magnetresonanzmethoden
}

\author{
René Botnar, Markus Beat Scheidegger, Peter Boesiger
}

\begin{abstract}
Institut für Blomedizinlsch Technlk und MedizIn/sche Informatlk der Unlvers/tat Zurlch und der Eldgenössischen Techn/schen Hochschule Zürich, Schwelz
\end{abstract}

\section{EINLEITUNG:}

Die Funktionen des Liquor cerebrospinalis sind zum einem der Schutz des zentralen Nervensystems rsp. die Regulation des intacranialen Drucks und zum anderen die Versorgung des Gehirns mit Năhrstoffen und Hormonen. Der Liquor cerebrospinalis wird vom choroideus plexus der beiden Seitenventrikel, des III. Ventrikels und des IV. Ventrikels gebildet. Er fliesst aus den Seitenventrikeln durch das Foramen interventriculare in den III. Ventrikel und von dort aus durch den Aquaeductus cerebri in den IV. Ventrikel. Aus dem IV. Ventrikel tritt der Liquor über die Foramina Luschkae rsp. das Foramen Magendii in den ausseren Liquorraum aus. Dort wird ein kleiner Teil in den Arachnoidalzotten des Subarachnoidalraums absorbiert.

Ein für das Liquorsystem typisches Krankheitsbild ist der Hydrocephalus, der eine abnormalen Zunahme des Liquorvolumens im Schädel beschreibt. Sie kann entweder durch eine Liquoruberproduktion im choroideus plexus oder durch eine tumorbedingte Verengung (Stenose) eines Liquorkanals (Aquaeductus, Foramen interventriculare) hervorgerufen werden. Herkömmliche Untersuchungsmethoden zur Liquorproduktionsmessung oder zur Darstellung der Liquorrăume (CT, nukleare Cisternographie) arbeiten entweder mit ionisierender Strahlung oder sind invasiv. Die Magnetresonanztomographie dagegen stellt eine vollig nicht invasive Untersuchungsmethode dar, die es ermoglicht sowohl die Anatomie der Liquorrăume darzustellen als auch den Fluss durch diese zu messen. In dieser Untersuchung sollte demonstriert werden, dass es mit MR-Methoden moglich ist den pulsatilen Liquorfluss sowohl zeitlich als auch räumlich aufgelost zu messen und daraus die Liquorproduktionsrate zu bestimmen. Hierzu wurden im Aquaeductus cerbri die Momentanflusswerte in ăquidistanten Zeitabstănden von $30 \mathrm{~ms}$ wăhrend des gesamten RR-Intervalls gemessen. Die Integration über die einzelnen Messwerte lieferte den Nettofluss und somit eiene Schătzwert für die tăgliche Produktionsrate des Liquors in den drei inneren

\section{Ventrikeln.}

\section{METHODE:}

Zur Erfassung der herzschlagbedingt pulsatilen Bewegung des Liquors wurde eine EKG getriggerte Phasen-Kontrast Sequenz benutzt, wobei die Geschwindigkeitskodierung in axiale Richtung erfolgte. Um eine Unterschatzung der Flussrate auf Grund einer nicht exakten $90^{\circ}$-Angulation zwischen Messschicht und Verlauf des Aquadductus gering zu halten und um den partial volume Effekt, der durch den gewundenen Verlauf des Aquáductus verursacht werden kann zu minimieren wurden die Schichten moglichst dünn $(5 \mathrm{~mm})$ gewahlt und immer so gelegt, dass der Verlauf des Aquaeductus innnerhalb der Schicht geradlinig war. Dies war am ehesten, wie in Figur 1 angedeutet, in der Einmündung des Aquaeductus in den 4 . Ventrikel gewăhrleistet.

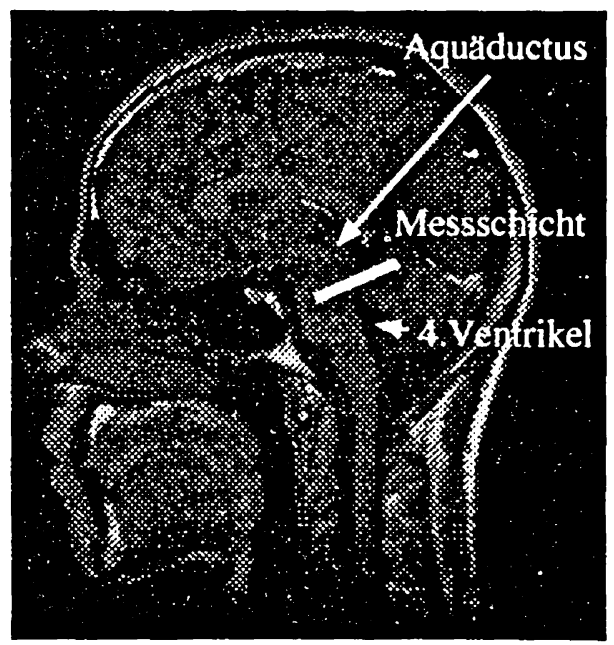

Fig 1: sagittales Spin-Echo Bild zur Planung der Messschicht.

Um eine gute Geschwindigkeitsauflosung zu erhalten wurde diese an den physiologischen Geschwindigkeitsbereich des Liquors an der Messstelle angepasst. Im Aquaeductus entsprach dies $62.8 \mathrm{rad} * \mathrm{~s} / \mathrm{m}$ und im intraspinalen Liquorraum auf der Hohe der Halswirbel $\mathrm{Cl} / \mathrm{C2} 20.9 \mathrm{rad} * \mathrm{~s} / \mathrm{m}$. Alle Untersuchungen wurden mit einem Philips Gyroscan S15/ACS II System unter Verwendung der Kopfspule durchge- 
fuhrt. Die schnelle Liquorflussănderung wăhrend der Systole erforderte eine Zeitaufosung von $30 \mathrm{~ms}$, was zu ungefăhr 30 Momentanflussbildern pro RRIntervall führte. Um das gesamte RR-Intervall wăhrend der Messung zu erfassen begann die Datennahme nur jeden 2.Herzschlag mit einer Verzögerungszeit von $8 \mathrm{~ms}$ auf die R-Zacke. Die gesamte Messdauer betrug ungefahr 9:25min (70Schlăge/Minute).

Die Genauigkeit der Produktionsratenmessung hăngt vorwiegend von der Nullphasenbestimmung (Phase des stationăren Gewebes) und von der Korrektur der Wirbelstromartefakte ab. Da die Geschwindigkeit der Spins proportional zur Phase $\phi=\gamma^{*} \mathrm{~B}_{0} * \mathrm{~T}+/-$ $\gamma^{*} v^{*} M_{1}$ des empfangenen Signals ist $\left(M_{1}: 1\right.$.Moment des Selektionsgradienten, v: Geschwindigkeit) wurden zwei Messungen mit betragsmässig gleichem jedoch einmal positivem und das andere $\mathrm{Mal}$ negativem 1. Moment durchgeführt. Die Differenz der beiden daraus erhaltenen Phasenbilder lieferte die Phasenwerte $\phi=2 * \gamma^{*} v^{*} \mathrm{M}_{1}+\phi_{\mathrm{Fehler}}$ der lokalen Liquorgeschwindigkeiten rsp. die des statischen $\mathrm{Ge}-$ webes plus lokale Phasenfehler, die von den unterschiedlichen Wirbelstromen dieser beiden Messungen resultierten. Um diese lokalen Phasenfehler zu korrigieren wurde ein spezieller auf unserem Gerăt verfügbarer Phasenfilter verwendet. Nach dieser Korrektur wurde der Nullphasenwert, der von den Phasenwerten des Aquâductus subtrahiert werden musste, in einem Gebiet mit statischen Gewebe bestimmt. Die danach erhaltenen Phasenwerte entsprachen den lokalen Flussgeschwindigkeiten im Aquăductus.

\section{Ergebnisse:}

In dieser Studie wurden 4 gesunde Probanden untersucht. Der Verlauf der Flusskurven im Aquaductus zeigt bei allen Probanden einen charakteristischen Verlauf. Ungefahr $100 \mathrm{~ms}$ nach der R-Zacke ist der Fluss maximal in craniale Richung. Darauf folgt eine schnelle Umkehr in caudale Richtung mit einen Flussmaximum bei ungefahr $35 \%$ des Herzintervalls. Im subarachnoidalen Raum auf $\mathrm{Cl} / \mathrm{C} 2$ Niveau ist die Flussumkehr von cranialer in caudale Richtung deutlich schneller als im Aquaeductus. Der maximale caudale Fluss tritt hier schon nach $20 \%$ des RRIntervalls auf. Die Zeitverschiebung zwischen dem maximalen Fluss caudalwärts im Aquaductus und dem auf der Hohe C1/C2 lieferte ein Liquorpulswellengeschwindigkeit von ungefahr $120 \mathrm{~mm} / \mathrm{s}$. Die Flussprofile im Aquaductus sind symmetrisch, wobei auf der Hohe $\mathrm{Cl} / \mathrm{C} 2$ eine deutliche anterior-posterior
Asymmmetrie zu erkennen ist.

Die Werte für die Liquorproduktionsrate liegen zwischen $750 \mathrm{ml} / \mathrm{Tag}$ und ungefăhr $1500 \mathrm{ml} / \mathrm{Tag} \mathrm{mit}$ einem Mittelwert von $(1063+/-410) \mathrm{ml} / \mathrm{Tag}$. Dieser Wert liegt uber dem Wert $500 \mathrm{ml} / \mathrm{Tag}$, der mit radioaktiven Tracern [1] gemessen wurden, jedoch innerhalb der Fehler bei dem Wert $900 \mathrm{ml} / \mathrm{Tag}$ der von einer anderen Gruppe [2] ebenfalls mit MRMethoden bestimmt wurde. Nachfolgend in Tabelle 1 sind die Ergebnisse der Liquorproduktionsratenbestimmung von drei Probanden aufgeführt.

\begin{tabular}{|l|l|l|l|}
\hline & $\begin{array}{c}\text { Produktions } \\
\text { rate } \\
\text { [mVday] }\end{array}$ & \multicolumn{1}{|c|}{$\begin{array}{c}\text { Fläche } \\
{\left[\mathrm{mm}^{2}\right]}\end{array}$} & $\begin{array}{c}\text { RR-Intervall } \\
{[\mathrm{ms}]}\end{array}$ \\
\hline Proband 1 & 921 & 6.42 & 878 \\
\hline Probend 2 & 743 & 9.87 & 818 \\
\hline Probend 3 & 1525 & 13.91 & 1118 \\
\hline
\end{tabular}

Tabelle 1: Liquorproduktionsraten

In der Figur 2 ist der zeitliche Verlauf des Flusses im Aquäductus uber das gesamte RR-Intervall dargestellt.

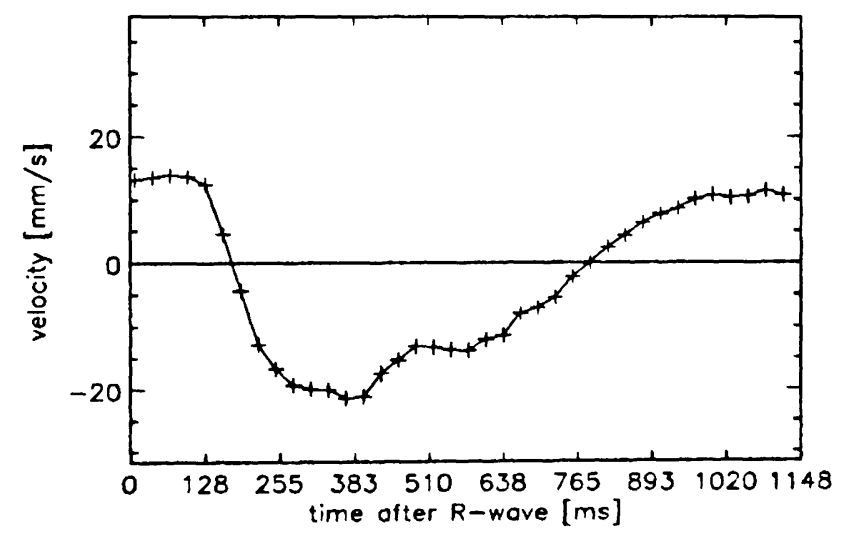

Figur 2: mittlere Geschwindigkeit des Liquors im Aquaductus cerebri.

Diskussion:

Die Phasenkontrast-Technik der MR-Tomographie ist eine geeignete nicht invasive Untersuchungsmethode sowohl zur Bestimmung der Liquorproduktion als auch zur Darstellung des Flusses in den Liquorrăumen. Sie kơnnte zB. zur Kontrolle der Liquorproduktion bei der Vergabe von produktionshemmenden oder stimulierenden Medikamenten eingesetzt werden.

[1]R. Rubin et al. , J. Neurosurg. 25: 430:436,1966

[2]C.T. Thomsen et al., Radiology 177: 659665,1990 